\title{
Supporting Information: Spontaneous Symmetry Breaking in Cyclo[18]Carbon
}

\author{
Zenner S. Pereira ${ }^{\dagger}$ and Edison Z. da Silva*,‡ \\ †Departamento de Ciência e Tecnologia, Universidade Federal Rural do Semi-Árido \\ (UFERSA), Campus Caraúbas, Rio Grande do Norte, Brazil \\ $\ddagger$ Institute of Physics "Gleb Wataghin”, UNICAMP, CP 6165, 13083-9859, Campinas - SP, \\ Brazil \\ E-mail: zacarias@ifi.unicamp.br
}




\section{Details of the Second Order Jahn-Teller Effect (SOJTE)}

Spontaneous symmetry breaking (SSB) is more frequent in nature than we imagine. Symmetry breaking in polyatomic systems is related to the breaking of degeneracy or the interaction of non degenerate states leading to symmetry changes in the system and its electronic structure. ${ }^{1}$ The pioneers in addressing these questions were Jahn and Teller. ${ }^{2}$ They first proposed the symmetry breaking to be due to the interaction of degenerate states, the phrase "Nature avoids degenerate states" can be clearly observed in elongated octahedral compounds widely studied in chemistry, as examples of distortions due to degeneracy breaking. ${ }^{3}$ Symmetry breaking in polyatomic systems is not limited to degenerate states, it can occur in nondegenerate states and brings consequences to the polyatomic system's topology. The Jahn-Teller effect as presented originally have been widely studied and new presentation forms were proposed. To this end Pearson ${ }^{4}$ have detailed the interaction of ground state and excited state, and this interaction is called Second-Order Jahn-Teller effect (SOJTE) and is grounded in symmetry and perturbation theory.

Two important expressions are needed to understand the SOJTE. As discussed by Jahn

and Teller ${ }^{2}$ and further discussed clearly by Pearson, ${ }^{4}$ after a distortion in the high symmetry structure, the energy of an electronic state can be written as

$$
E=E_{0}+\left\langle\psi_{0}\left|\frac{\partial U}{\partial Q}\right| \psi_{0}\right\rangle Q+\left\langle\psi_{0}\left|\frac{\partial^{2} U}{\partial Q^{2}}\right| \psi_{0}\right\rangle \frac{Q^{2}}{2}+\sum_{k} \frac{\left[\left\langle\psi_{0}\left|\frac{\partial U}{\partial Q}\right| \psi_{k}\right\rangle Q\right]^{2}}{E_{0}-E_{k}}
$$

The associated new eigenfuctions of the distorted configuration can be writen a;

$$
\psi=\psi_{0}+\sum_{k} \frac{\left\langle\psi_{0}\left|\frac{\partial U}{\partial Q}\right| \psi_{k}\right\rangle Q}{E_{0}-E_{k}}
$$

In equations (1) and (2), $U$ represent the interaction energy of atoms and nuclei, $\psi_{0}$ represents a ground state eigenfunction associated with the energy $E_{0}, \psi_{k}$ is a excited state eigenfunction with energy level $E_{k} . \mathrm{Q}$ is the reaction coordinate which is small within this 
approximation. Equations (1) and (2) are thus, understood in terms of a distortion of the high symmetry to the less symmetric structure. As a consequence, energies and eigenfunctions of the symmetric (no distortion) are modified resulting in the energy $E$ (eq.1) and new eigenfunction $\psi$ (eq.2). In eq.1, the second and third terms are first order perturbations, while the third is of second order, due to changes in the electronic structure, it is called transition density. The SOJT effect is a consequence of the Q quadratic terms in eq.1. The third term is always positive when the structure is distorted, while the last term is always negative and depends on the mixing of ground and excited states. This interaction occurs if the energy gap between ground and excited states is not large and also that the $\psi_{0}$ and $\psi_{k}$ have the same symmetry. ${ }^{4,5}$

When the ground state eigenfuntion is strongly affected by the interaction with the excited state, the last term of eq.1 dominates the third term effecting a downward concavity in the ExQ diagram, where $\mathrm{E}$ is the energy and $\mathrm{Q}$ the a reaction coordinate associated to the direction in which the structure change occurs. This behavior of the electronic states can be better understood in the formulation presented by Besusker. ${ }^{1}$ Besusker presents a more general and detailed study of the ground state excited state interaction analyzing the curvature $(\mathrm{K})$ of the adiabatic potential energy surface (APES) and the coupling of the electronic structure and the nuclei. The curvature $\mathrm{K}$ in a particular direction (reaction coordinate direction) can be written as:

$$
K=\left\langle\psi_{0}\left|\frac{\partial^{2} H}{\partial Q^{2}}\right| \psi_{0}\right\rangle-2 \sum_{k} \frac{\left|\left\langle\psi_{0}\left|\frac{\partial H}{\partial Q}\right| \psi_{k}\right\rangle\right|^{2}}{E_{k}-E_{0}}
$$

Where $\mathrm{H}$ is the Hamiltonian of the system and we can write $K=K_{0}+K_{v}$ with:

$$
K_{0}=\left\langle\psi_{0}\left|\frac{\partial^{2} H}{\partial Q^{2}}\right| \psi_{0}\right\rangle
$$




$$
K_{v}=-2 \sum_{k} \frac{\left|\left\langle\psi_{0}\left|\frac{\partial H}{\partial Q}\right| \psi_{k}\right\rangle\right|^{2}}{E_{k}-E_{0}}
$$

Note that the Hamiltonian is expanded from the high symmetry structure configuration, in terms of the distortion, the reaction coordinate. Since $K_{0}>0,{ }^{1,6} K_{v}$ is therefore the only source of instability that leads to the symmetry breaking in polyatomic systems. Therefore, in such systems, in order to achieve SSB is mandatory interaction of the ground state interaction and excited states. This interaction is not limited to the HOMO LUMO or among only two states and it follows selection rules. For simplicity and considering only the interaction of two states, one can obtain the characteristic curve for the energies of the excited and ground state energies ${ }^{1}$ as:

$$
\epsilon_{ \pm}=\frac{1}{2} K_{0} Q^{2} \pm \frac{F^{2}}{\Delta} Q^{2} \pm \Delta \mp \frac{1}{4} \frac{F^{4}}{\Delta^{3}} Q^{4} \pm \ldots
$$

Where $\epsilon_{+}$represents the excited interacting state energy level and $\epsilon_{-}$the ground interacting state energy level. $Q$ is the reaction coordinate along the symmetry breaking occurs. $2 \Delta=E_{k}-E_{0}$ e $F=\left\langle\psi_{0}|\partial H / \partial Q| \psi_{k}\right\rangle$. The term $F$ denotes the excited state interaction with the ground state and is the important parameter for the effect. It is clear that when excited state - ground state interaction occurs $\left(F^{2}>0\right)$, the excited state increases its energy while the ground state lowers its energy. Therefore, an energetic competition is stablished among the state involved in the interaction with the ones not involved by the interaction. As a consequence of this competition the structure finds a new equilibrium in a less symmetric state. When there is no excited state - ground state interaction, or this interaction is weak, the value of $F$ is zero or close to it, in such cases, the upward concavity term dominates and the system stays in the high symmetry configuration. 


\section{STM images and Molecular orbitals}

Using the Tersorf e Hamann ${ }^{7}$ formulation we constructed simulated STM images from the local density of states (LDOS) of both $\mathrm{C}$ and $\mathrm{P}$ structures. Ths formulation considers an approximation where isosurface calculations taken at some height in $\mathrm{z}$ direction, measured from the center of the molecule gives the effect similar to the STM with point like resolution. Figure S1 depicts the colored LDOS taken from $\mathrm{z}=0$ for the polyynic and cumulenic structures. The polyynic structure shows short bonds with high LDOS, a clear evidence of one $\sigma$ and two $\pi$ bonds, the $\sigma$ bond which is frontal and stronger than the lateral $\pi$ bonds. The long bond with smaller LDOS is due to a single $\sigma$ bond. In comparison, the unstable cumulenic structure shows equally spaced and similar bonds, evidence of a double bond formed by a sigma and a $\pi$ bond. In this work we show that cumulenic structure is unstable, that there is a coupling of the electronic structure and the nuclei positions, an interaction of ground state with excited states, responsible for the symmetry breaking of the cumulenic $\mathrm{D}_{18 h}$ structure going to the stable polyynic $\mathrm{D}_{9 h}$ structure.

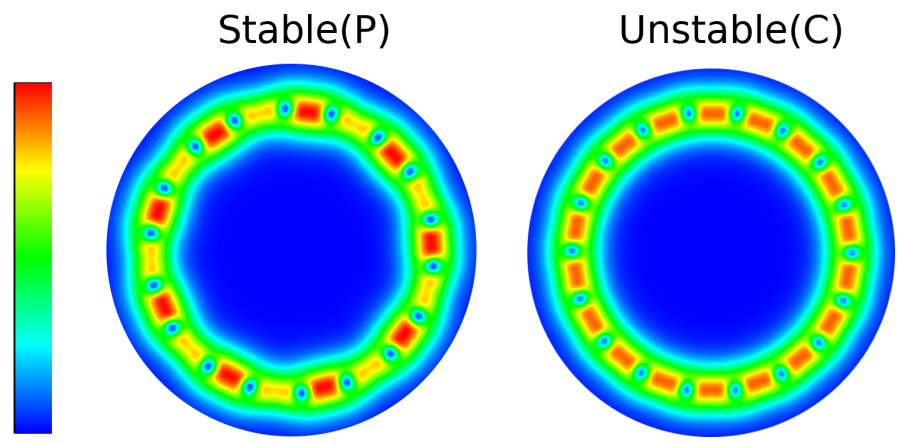

Figure S 1: Simulated STM, the color scale is normalized, red is high intensity and blue is low intensity. The slice represents the isodensity taken at $\mathrm{z}=0$, taking the center of the structure as reference. Taking the Fermi level in the middle of the HOMO-LUMO (-5.01, $\mathrm{eV})$, bias volt taken as $-25.6 \mathrm{eV}$ bellow the Fermi level

Figure S2 shows simulated STM images in gray scale, similar to Fig. S1 for different bias voltages with $\mathrm{z}=0.3 \AA$. As the bias is decreasing (negative values to bias voltage in $\mathrm{eV}$ ) more states are involved in the LDOS and the total effect is closer to the results previously presented by Kaiser. $^{8}$ 

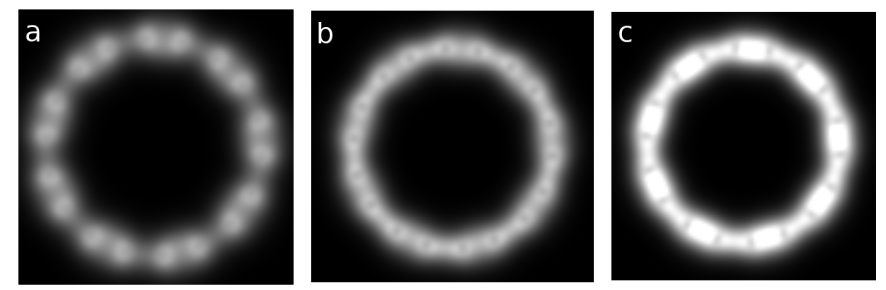

Figure S 2: Simulated STM, the grey scale is normalized, white denotes high intensity and black is null intensity. The slice represents the isodensity taken at $\mathrm{z}=0.3 \AA$, taking the center of the structure as reference. Taking the Fermi level in the middle of the HOMO LUMO (-5.01 eV), The bias voltage was taken at values (a) $-17.0 \mathrm{eV}$, (b) $-22.18 \mathrm{eV}$ and (c) $-25.6 \mathrm{eV}$.

Figure S3 shows a comparison of the MO of both structures, namely, cumulenic and polyynic. It is observed that in the polyynic form the frontier orbitals show symmetry distortions. This effect occurs until HOMO-3, they do not show in HOMO-4 an deeper MOs and this was verified in the energy versus $Q_{\theta}$. The cumulenic structure on the other hand, shows symmetry even for the frontier orbitals. The high symmetry guarantees the equilibrium in the probability density although being an unstable equilibrium. The deeper states of both structures have similar MOs.

Figure S4 shows once again the energy diagram with the comulenic structure at the top unstable maximum and at the two minima, the two possible results of the evolution driven by SOTJE. The carbon atoms highlighted in red, are enlarged to show the triple (T) bond and the Single $(\mathrm{S})$ bonds that alternate in the pollyynic structute 


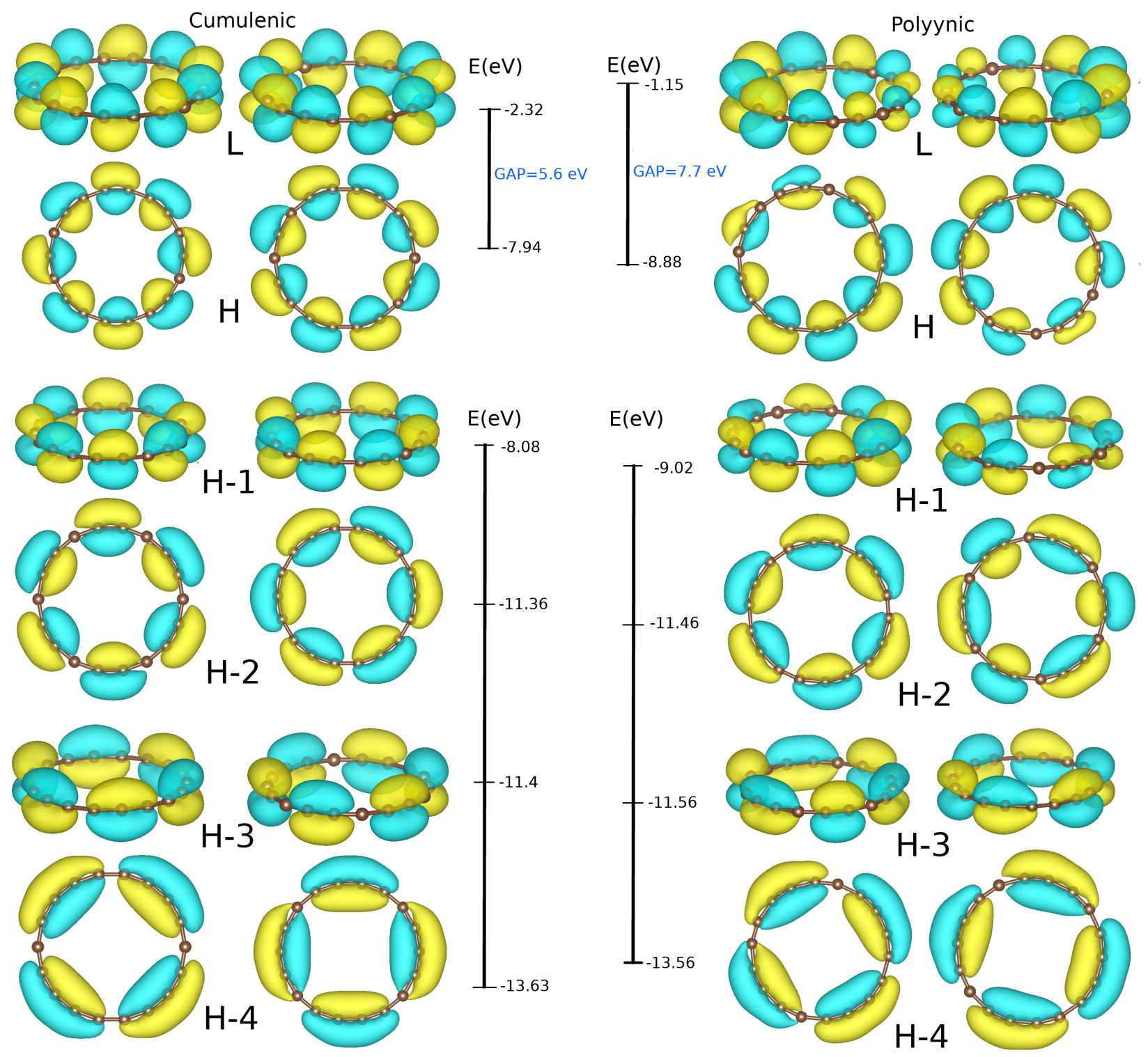

Figure S 3: Molecular Orbitals (MO) of the polyynic and cumulenic structures and they respective gaps. 


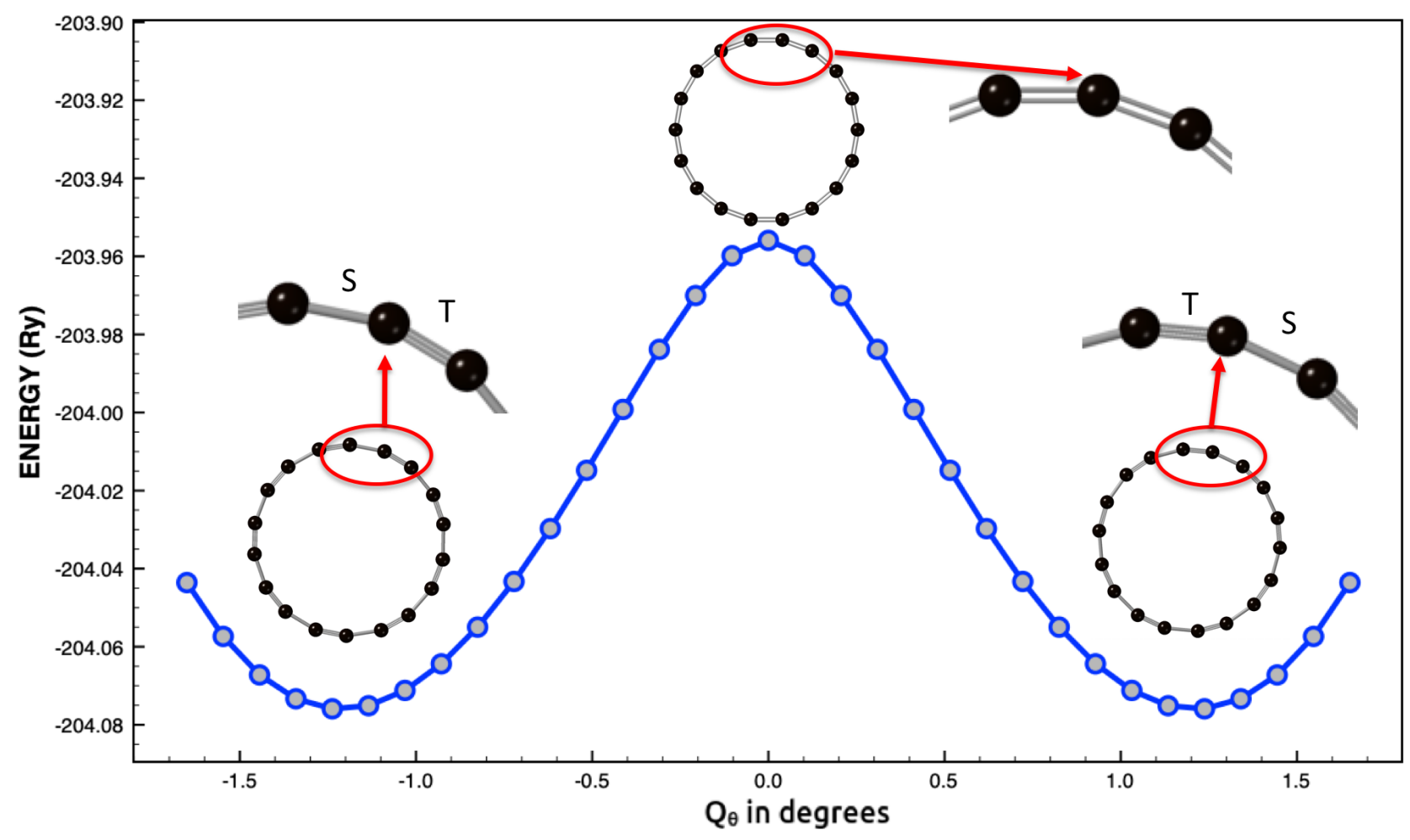

Figure S 4: The two possible pollynic struvture, result of the SOJTE.

\section{References}

(1) Bersuker, I. B. Pseudo-Jahn-Teller Effect-A Two-State Paradigm in Formation, Deformation, and Transformation of Molecular Systems and Solids. Chem. Rev. 2013, 113, 1351-1390.

(2) H. A. Jahn, E. T.; George, D. F. Stability of polyatomic molecules in degenerate electronic states I - Orbital degeneracy. Proceedings of the Royal Society A 1937, 161, 220-235.

(3) Halcrow, M. A. Jahn-Teller distortions in transition metal compounds, and their importance in functional molecular and inorganic materials. Chem. Soc. Rev. 2013, 42, 1784-1795.

(4) Pearson, R. G. The second-order Jahn-Teller effect. Journal of Molecular Structure: 
Theochem 1983, 103, $25-34$.

(5) Pearson, R. G. Concerning Jahn-Teller Effects. Proceedings of the National Academy of Sciences 1975, 72, 2104-2106.

(6) Bersuker, I. B. On the origin of dynamic instability of molecular systems. Theoretica chimica acta 1984, 66, 161-172.

(7) Tersoff, J.; Hamann, D. R. Theory of the scanning tunneling microscope. Phys. Rev. B 1985, 31, 805-813.

(8) Kaiser, K.; Scriven, L. M.; Schulz, F.; Gawel, P.; Gross, L.; Anderson, H. L. An sphybridized molecular carbon allotrope, cyclo[18]carbon. Science 2019, 365, 1299-1301. 